\title{
Residence Time Prediction of Type 1 and 2 Kinase Inhibitors from Unbinding Simulations.
}

\author{
Abdennour Braka ${ }^{[\mathrm{a}, \mathrm{b}]}$, Norbert Garnier ${ }^{[\mathrm{b}]}$, Pascal Bonnet ${ }^{[\mathrm{a}]}$, Samia Aci-Sèche ${ }^{{ }^{[\mathrm{a}]}}$ \\ [a] Institut de Chimie Organique et Analytique (ICOA), UMR7311 Université d'Orléans-CNRS, Rue de Chartres - BP 6759, \\ 45067 Orléans Cedex 2 France \\ [b] Centre de Biophysique Moléculaire (CBM) UPR 4301, CNRS, Rue Charles Sadron, 45071 Orléans Cedex 2, France \\ KEYWORDS: Drug design $\bullet$ Kinetics $\bullet$ Molecular dynamics $\bullet$ Residence time $\bullet$ Transition state theory
}

\begin{abstract}
Residence time is an important parameter associated to in vivo drug's safety and efficacy. The determination or modulation of kinetic rates correlated to residence time is performed to identify the best drug candidates in an early stage of drug design project. Herein, we developed a novel computational methodology based on molecular dynamics simulations and transition state theory in order to predict ligand unbinding pathways, kinetic rates and residence times. The studied set was composed of eight ligands with fast, intermediate and slow dissociation rates and binding to the active and inactive states of p38 $\alpha$ protein kinase. The proposed method provides an excellent correlation between the predicted values and the experimentally measured kinetic rates, in addition to a detailed characterization of the kinetic paths at the atomic level.
\end{abstract}

The drug candidate optimization and selection processes are often based on equilibrium thermodynamic constants such as the dissociation constant $\left(\mathrm{K}_{\mathrm{D}}\right)$ and the half maximal inhibitory concentration $\left(\mathrm{IC}_{50}\right)$. Nonetheless, a high number of molecules still fails in phase II clinical trials during the evaluation of their efficacy in humans[1], resulting in high attrition rates and $\mathrm{R} \& \mathrm{D}$ costs. Indeed, in an in vivo delivery system, the dynamic of distribution and excretion modifies regularly the availability of a drug for binding to its target. This biological phenomenon requires predictive parameters that take into account the dynamic behavior of ligand binding to increase the success rate in clinical trials. In the last decade, binding kinetic constants $\left(\mathrm{k}_{\mathrm{on}}\right.$ and $\left.\mathrm{k}_{\mathrm{off}}\right)$ and in particular residence time (RT), emerged as good indicators of the in vivo efficacy.[2,3] Highlighted in 2004 by Swinney and introduced thereafter in 2006 by Copeland et al,[4, 5] RT ( $\left.1 / \mathrm{k}_{\text {off }}\right)$, which reflects the lifetime of a drug-target complex, significantly influences pharmacodynamics. So, RT-based ligand optimization may strongly improve drug's selectivity, efficacy and safety.[6,7]

Nowadays, a number of experimental methods are available to measure protein-ligand binding kinetics, as technologies using a labeled molecule such as fluorescence labeling, radioligand binding assays or stopped-flow technology, or label-free technologies such as surface plasmon resonance (SPR).[8] However, the high cost of these techniques does not allow their use to provide kinetics data for a large number of molecules. While many efforts are currently pursued in kinetics rate prediction, no efficient computational approach has still been developed to correctly estimate kinetics parameters. Nevertheless, recent studies have shown the possibility of simulating the association process of a ligand to a target with conventional molecular dynamics (cMD) for processes in the range of a few microseconds.[ 9,10] Despite the progress of computing power, cMD remains very expensive in computational time and simulating dissociation events in the range of minutes to hours is still challenging, even by including an artificial bias. Thus, the development of enhanced computational methods is strongly needed to simulate the proteinligand unbinding process and predict efficiently kinetics rate values and residence time.

Some efforts have recently been carried out in this direction. We could mention for instance the estimation of the dissociation rate of the benzamidine-trypsin complex by adaptive multilevel splitting method and metadynamics[11,12] or the one of a fragment of Doramapimod-p38 $\alpha$ complex by metadynamics[13] leading to RTs in good agreement with the experimental values. However, these methods were applied only on one ligand and have some limitations in the choice of the metadynamic collective variables. In another study, the RTs of seven inhibitors of glucokinase GK1 was estimated by scaled MD.[14] These predicted RTs were directly deduced from simulation times and the energetic profiles of transition states were omitted. Finally, in all those studies, inhibitors have RTs in the range of several seconds while longer RTs are desired for many drugs in clinical trials.

In this work, we developed and applied a novel method to predict RT on $\mathrm{p} 38 \alpha$ mitogen-activated protein kinases. This protein kinase plays a key role in regulating the proinflammatory cytokines biosynthesis and is a therapeutic target for the treatment of autoimmune and inflammatory diseases.[15] Like most protein kinases, p38 $\alpha$ exists in two major conformational states: an active state (DFG-in) in which the ligands bind in the ATP opened pocket and an inactive state (DFG-out) in which a second hydrophobic pocket is opened through a conformational switch of the DFG motif.[16] The inhibitors targeting the active conformation of the protein kinase are called type I inhibitors and the ones binding the inactive form, type II inhibitors. Here, we explore the unbinding paths of eight p $38 \alpha$ kinase inhibitors, among them three are type I and five are type II. We also estimate the associated 
unbinding free energies by using bootstrapping and Weighted Histogram Analysis Method (WHAM) and the binding kinetic constant of the compounds with the help of transition state theory (TST).

To challenge the novel predictive approach, the choice of the ligands was based on three criteria: 1) ligands of different sizes such as fragments and ligands belonging to two different chemical series, 2) experimental residence times ranging from seconds to hours, 3) type I and II kinase inhibitors to evaluate the conformational state of the protein kinase (active or inactive) (Table 1, similarity matrix and chemical structures in Figure S1). Among the selected ligands, three of them, B96, BMU, SB6, are structurally solved in complex with p38 and available under PDB codes $1 \mathrm{KV} 2,1 \mathrm{KV} 1$ and $1 \mathrm{~A} 9 \mathrm{U}$ respectively. For the other ligands, the structure of each proteinligand complex was built by making minor substitutions of the most similar solved ligand in its crystallographic structure (SI.2.1, Table S1).

Table 1. Comparison of experimental affinities, kinetics, and protein conformation states of studied inhibitors

\begin{tabular}{|l|l|l|l|l|l|}
\hline $\begin{array}{l}\text { Ligand } \\
\text { a] }\end{array}$ & $\mathrm{K}_{\mathrm{D}}(\mathrm{M})$ & $\mathrm{K}_{\text {off }}\left(\mathrm{s}^{-1}\right)$ & $\begin{array}{l}\mathrm{RT} \\
\text { Exp. }\end{array}$ & $\begin{array}{l}\text { DFG } \\
\text { orien- } \\
\text { tation }\end{array}$ & $\begin{array}{l}\text { Experi- } \\
\text { mental } \\
\text { method } \\
\text { [reference }]\end{array}$ \\
\hline B96 & $6.1 \mathrm{e}-10$ & $5.2 \mathrm{e}-5$ & $5.3 \mathrm{~h}$ & Out & SPR $^{[17]}$ \\
\hline BMU & $1.1 \mathrm{e}-6$ & $2.8 \mathrm{e}-2$ & $35.7 \mathrm{~s}$ & Out & SPR $^{[17]}$ \\
\hline SB5 & $4.0 \mathrm{e}-8$ & $2.7 \mathrm{e}-2$ & $37 \mathrm{~s}$ & In & SPR $^{[17]}$ \\
\hline SB6 & $7.8 \mathrm{e}-8$ & $1.3 \mathrm{e}-1$ & $7.7 \mathrm{~s}$ & In & SPR $^{[17]}$ \\
\hline SB7 & $5.1 \mathrm{e}-8$ & $6.7 \mathrm{e}-2$ & $14.9 \mathrm{~s}$ & In & SPR $^{[17]}$ \\
\hline BR5 & $9.7 \mathrm{e}-11$ & $1.5 \mathrm{e}-5$ & $18.5 \mathrm{~h}$ & Out & SFF $^{[18]}$ \\
\hline BR8 & $2.3 \mathrm{e}-8$ & $3.3 \mathrm{e}-3$ & $5 \mathrm{~min}$ & Out & SFF $^{[18]}$ \\
\hline B12 & $1.6 \mathrm{e}-8$ & $2.6 \mathrm{e}-5$ & $10.6 \mathrm{~h}$ & Out & SFF $^{[18]}$ \\
\hline
\end{tabular}

[a] Structures reported in Figure S1., SPR: Surface Plasmon Resonance, SFF: Stopped-Flow Fluorescence

To investigate the unbinding process of each ligand, we simulated their behavior along the dissociation event, from the binding pocket up to the solvent, using steered molecular dynamics (SMD). We applied a biasing force to steer the ligand out from the binding site by increasing the distance between the center of mass of the ligand $\left(\mathrm{COM}_{\mathrm{Lig}}\right)$ and the center of mass of the binding site $\left(\mathrm{COM}_{\mathrm{BS}}\right)$ with a constant velocity of $1 \AA . n s^{-1}$. This unsupervised approach allows the ligand to find the exit path according to the encountered energetic barriers. Since the exploration of paths can change from one simulation to another, we performed ten replicates of simulations per ligand in order to sample a large number of events. This number of replicates is recommended to have a statistically meaningful estimation of kinetics.[19]
As expected, all replicates of type I inhibitors SB5, SB6 and SB7 select dissociation paths toward the solvent area side of the ATP pocket, since the allosteric pocket is not available in the DFG-in conformation. They all have short RTs. In the case of the type II inhibitors, for which two pockets exist, we observe two interesting exit paths. For the inhibitors with a long RT such as B12, B96 and BR5, dissociation pathways go through the solvent area side of the allosteric pocket in all replicates. In the case of the inhibitors BMU and BR8 having short and medium RT, five replicates present an exit path through the solvent area side of the ATP pocket and five through the allosteric pocket (Figure 1 (a)). These observations show that short and long RTs are mainly induced by ATP and allosteric channels respectively. To estimate the free energy of dissociation with a reasonable statistical relevance, we considered the ten replicates for each ligand, including those taking two different paths.

Using biased molecular dynamics simulations such as SMD, the free energy profile is obtained as a function of a reaction coordinate. This energy profile is referred to potential of mean force (PMF). The PMF is usually calculated from the work of the steering force using the Jarzynski equality, which allows to compute free energy differences.[20] If the sampling is insufficient, the simulations have to be restarted with longer velocities. However, evaluating the quality of sampling to obtain a relevant estimation remains difficult with this method. To solve this issue, we first applied a bootstrapping approach where each path is split, according to the reaction coordinate, into small windows of $0.5 \AA$. Then, we generated from the centroid of each window a new statistically robust distribution (SI.2.5, Figure S3) by performing $5 \mathrm{~ns}$ of resampling with the same parameters as those used in the SMD method, leading to a total of $450 \mathrm{~ns}$ per replicate and of $4.5 \mu \mathrm{s}$ for each ligand. Then, the PMF was rebuilt from the new probability distributions using WHAM. [21] (Figure 1 (b) for BMU, Figure S4 for all ligands).

Our estimation of free energies was motivated by the BellEvans theory of binding kinetics,[22-23] which is supported by previous experimental studies showing a correlation between the height of the energy barriers (E) and the measured $\mathrm{k}_{\text {off }}$.[24-25] This correlation follows an Arrhenius law where $\mathrm{A}$ is the pre-exponential factor, $\mathrm{kB}$ is the Boltzmann constant and $\mathrm{T}$ is the temperature [Eq. (1)].

$$
\mathrm{k}=\mathrm{A} \cdot \mathrm{e}^{-E / \mathrm{k}_{\mathrm{B}} \mathrm{T}}
$$

Assuming that the dissociation events of the studied kinase inhibitors follow an Arrhenius like behavior, the estimation of their activation free energies, which correspond to the energy barriers $\mathrm{E}$ in Eq. (1), can be used to calculate the dissociation rate $\left(\mathrm{k}_{\mathrm{off}}\right)$. For this purpose, the transition state theory (TST) is used according to Eq. (2), where $\Delta \mathrm{G}_{\text {off }}$ is the absolute unbinding free energy of activation.

$$
\mathrm{k}_{\mathrm{off}}=\mathrm{A} \cdot \mathrm{e}^{-\Delta \mathrm{G}_{\mathrm{off}} / \mathrm{k}_{\mathrm{B}} T}
$$

Another variant of Eq. (2) can be written by using the logarithm and the equation above is in the form $y=a x+b$ [Eq. (3)].

$$
-\log \mathrm{k}_{\mathrm{off}}=\Delta \mathrm{G}_{\mathrm{off}} / \mathrm{k}_{\mathrm{B}} \mathrm{T}-\log \mathrm{A}
$$


$\mathrm{k}_{\text {off }}$ can be calculated from the determination of the preexponential factor (A) and the slope from a linear correlation.. We estimated $\Delta \mathrm{G}_{\text {off }}$ in a context of a kinetic model having two energetic states (bound and unbound states) and by considering that the resampled replicates explore the transition states and describe the rare events between these two states. This activation free energy was calculated as the $\Delta \mathrm{G}_{\text {off }}$ 's average of the ten replicates for each inhibitor $\left(\left\langle\Delta \mathrm{G}_{\mathrm{off}}\right\rangle\right)$ (table 2$)$.

Figure 1. Unbinding simulation results for the ligand BMU: (a) ATP pocket path from replicate 3 and allosteric pocket path from
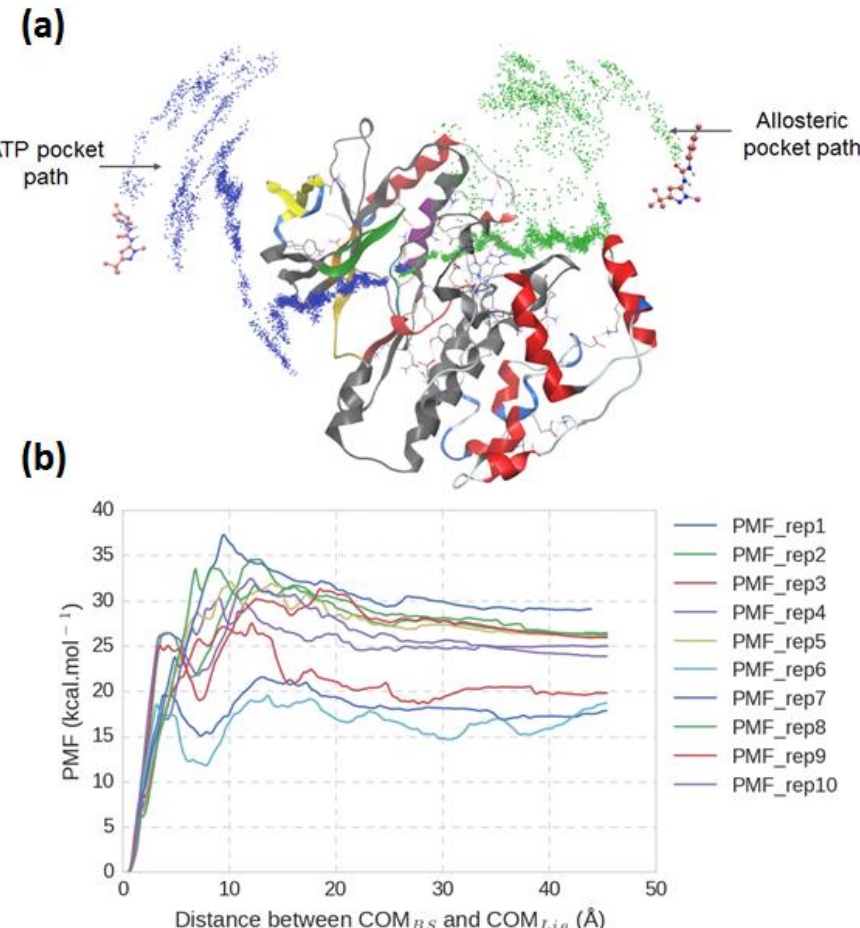

replicate 9. Each point represents the position of the $\mathrm{COM}_{\mathrm{Lig}}$ at each step of the simulation. (b) Potential of Mean Force (PMF) built from bootstrapping of each replicate.

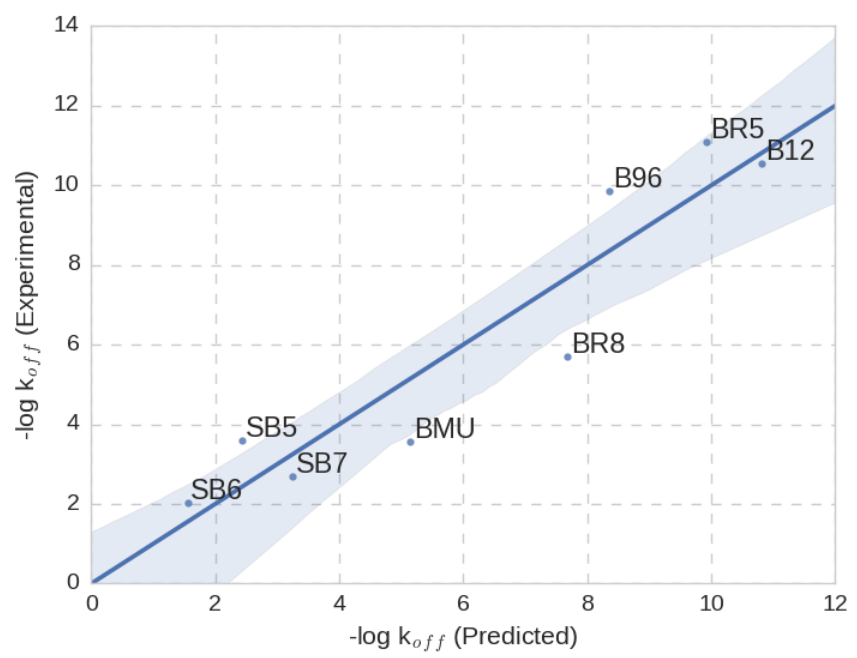

Figure 2. The correlation between the experimental and predicted $\mathrm{k}_{\text {offs. }}$.
The linear regression analysis between the estimated $\left\langle\Delta \mathrm{G}_{\text {off }}\right\rangle$ and the experimental $\mathrm{k}_{\text {off }}$ values disclose a very strong positive correlation with a coefficient of determination $\mathrm{r}^{2}$ of 0.88 and a good confidence interval (Figure S5). The linear regression equation $(\mathrm{y}=0.26 \mathrm{x}-1.11)$ was used to calculate the predicted $\mathrm{k}_{\text {offs }}$, and RTs were deduced from these predicted $\mathrm{k}_{\text {offs }}$ (Figure 2 , Table 2$)$. It should be noted that a weak correlation $\left(r^{2}=0.4\right)$ is obtained when the linear regression analysis is made between the estimated $\langle\Delta$ Goff $\rangle$ and the experimental affinity values $\mathrm{K}_{\mathrm{D}}$ (Figure $\mathrm{S} 6$ ).

Table 2. Comparison of Experimental RT, Predicted RT and average of relative activation free energy $\left\langle\Delta \mathbf{G}_{\text {off }}\right\rangle$.

\begin{tabular}{|l|l|l|l|}
\hline Ligand & $\langle\Delta$ Goff $\rangle\left(\mathrm{kcal}^{\mathrm{mol}}{ }^{-1}\right)$ & RT Pred & RT Exp \\
\hline B96 & 36.1 & $1.2 \mathrm{~h}$ & $5.3 \mathrm{~h}$ \\
\hline BMU & 23.8 & $2.8 \mathrm{~min}$ & $35.7 \mathrm{~s}$ \\
\hline SB5 & 13.5 & $11 \mathrm{~s}$ & $37 \mathrm{~s}$ \\
\hline SB6 & 10.2 & $4 \mathrm{~s}$ & $7.7 \mathrm{~s}$ \\
\hline SB7 & 16.6 & $25 \mathrm{~s}$ & $14.9 \mathrm{~s}$ \\
\hline BR5 & 42.1 & $5.7 \mathrm{~h}$ & $18.5 \mathrm{~h}$ \\
\hline BR8 & 33.6 & $36 \mathrm{~min}$ & $5 \mathrm{~min}$ \\
\hline B12 & 45.5 & $13.9 \mathrm{~h}$ & $10.6 \mathrm{~h}$ \\
\hline
\end{tabular}

The proposed method ranks most inhibitors according to their experimental RTs and in respect of their time scales (seconds, minutes, hours). These results are very encouraging and show that it is possible to apply molecular dynamics simulations to predict the kinetics parameters of inhibitors with long RTs and with medium and short RTs. However, the accuracy of the predictive power of the method is sensitive to reliable experimental values, to the heterogeneity of assay conditions or to the kinetic behaviour, for which the Arrhenius linearity is not always perfect. While the predicted $\mathrm{k}_{\text {off }}$ values obtained here are in good agreement with experimental values, these parameters have to be taken into account in future studies to improve the method.

In summary, we explored at the atomic level the mechanism of dissociation of eight type I and II kinase inhibitors having long, medium short RTs. We first demonstrated that the RT scale depends on the path followed by the ligand, from the ATP pocket side or from the allosteric pocket side for inhibitors with short or long RTs respectively. These results provide new insights in the optimization of $\mathrm{p} 38 \alpha$ inhibitors. If the goal is to increase the RT, the synthesis of the molecules should be guided so that the ligand dissociation from the allosteric side is favored, according to the interactions characterized at the atomic level (Video SI.4). Then, we estimated the activation free energies for each inhibitor from the simulated dissociation paths. With the help of a linear regression analysis, we show an excellent correlation between calculated energies and measured $\mathrm{k}_{\text {off }}$. This means that the dissociation process follows an Arrhenius-like behaviour, irrespective of the receptor's conformation states. This linear regression model was used to predict RTs of eight inhibitors using the transition state theory. Using an original computational approach, we were able to 
clearly differentiate the protein kinase inhibitors with short, medium and long RTs. However, further studies are needed to improve the absolute values of the predicted RT and to validate the method on other therapeutic targets.

\section{ASSOCIATED CONTENT}

\section{Supporting Information.}

Biological data of the inhibitors, details on computational method, computing time, Movie of unbinding process of B96 from one replicate, Tables S1-S2, and Figures S1-S6.

This material is available free of charge via the Internet at http://pubs.acs.org.

\section{AUTHOR INFORMATION}

\section{Corresponding Author}

* samia.aci-seche@univ-orleans.fr

* pascal.bonnet@univ-orleans

\section{Funding Sources}

This work was supported by the French National Research Agency (ANR-13-JSV5-0001) and the Région Centre Val de Loire.

\section{ACKNOWLEDGMENT}

The authors wish to thank the Orléans-Tours CaSciModOT at the Centre de Calcul Scientique de la Région Centre Val de Loire and the Centre Régional Informatique et d'Applications Numériques de Normandie (CRIANN) for providing computer facilities.

\section{REFERENCES}

1. Hay, Michael, David W. Thomas, John L. Craighead, Celia Economides, and Jesse Rosenthal. 2014. "Clinical Development Success Rates for Investigational Drugs." Nature Biotechnology 32 (1): 40-51. https://doi.org/10.1038/nbt.2786.

2. Schuetz, Doris A., Wilhelmus Egbertus Arnout de Witte, Yin Cheong Wong, Bernhard Knasmueller, Lars Richter, Daria B. Kokh, S. Kashif Sadiq, et al. 2017. "Kinetics for Drug Discovery: An Industry-Driven Effort to Target Drug Residence Time." Drug Discovery Today 22 (6): 896-911. https://doi.org/10.1016/j.drudis.2017.02.002.

3. Copeland, Robert A. 2016. "The Drug-Target Residence Time Model: A 10-Year Retrospective." Nature Reviews. Drug Discovery 15 (2): 87-95. https://doi.org/10.1038/nrd.2015.18.

4. Swinney, David C. 2004. "Biochemical Mechanisms of Drug Action: What Does It Take for Success?" Nature Reviews. Drug Discovery 3 (9): 801-8. https://doi.org/10.1038/nrd1500.

5. Copeland, Robert A., David L. Pompliano, and Thomas D. Meek. 2006. "Drug-Target Residence Time and Its Implications for Lead Optimization." Nature Reviews. Drug Discovery 5 (9): 730-39. https://doi.org/10.1038/nrd2082.

6. Guo, Dong, Thea Mulder-Krieger, Adriaan P. IJzerman, and Laura H. Heitman. 2012. "Functional Efficacy of Adenosine $A_{2} \mathrm{~A}$ Receptor Agonists Is Positively Correlated to Their Receptor Residence Time." British Journal of Pharmacology 166 (6): 1846-59. https://doi.org/10.1111/j.1476-5381.2012.01897.x

7. Walkup, Grant K., Zhiping You, Philip L. Ross, Eleanor K. H. Allen, Fereidoon Daryaee, Michael R. Hale, John O'Donnell, et al. 2015. "Translating Slow-Binding Inhibition Kinetics into Cellular and in Vivo Effects." Nature Chemical Biology 11 (6): 416-23. https://doi.org/10.1038/nchembio.1796.

8. Zheng, Xiwei, Cong Bi, Zhao Li, Maria Podariu, and David S. Hage. 2015. "Analytical Methods for Kinetic Studies of Biological Interactions: A Review." Journal of Pharmaceutical and Biomedical Analysis $113 \quad$ (September): $163-80$. https://doi.org/10.1016/j.jpba.2015.01.042.

9. Shan, Yibing, Eric T. Kim, Michael P. Eastwood, Ron O. Dror, Markus A. Seeliger, and David E. Shaw. 2011. "How Does a Drug Molecule Find Its Target Binding Site?" Journal of the American
Chemical Society 133

https://doi.org/10.1021/ja202726y.

(24):

$9181-83$.

10. Pan, Albert C., Huafeng Xu, Timothy Palpant, and David E. Shaw. 2017. "Quantitative Characterization of the Binding and Unbinding of Millimolar Drug Fragments with Molecular Dynamics Simulations." Journal of Chemical Theory and Computation 13 (7): 3372-77. https://doi.org/10.1021/acs.jctc.7b00172.

11. Tiwary, Pratyush, Vittorio Limongelli, Matteo Salvalaglio, and Michele Parrinello. 2015. "Kinetics of Protein-ligand Unbinding: Predicting Pathways, Rates, and Rate-Limiting Steps." Proceedings of the National Academy of Sciences 112 (5): E386-E391. https://doi.org/10.1073/pnas.1424461112.

12. Teo, Ivan, Christopher G. Mayne, Klaus Schulten, and Tony Lelièvre. 2016. "Adaptive Multilevel Splitting Method for Molecular Dynamics Calculation of Benzamidine-Trypsin Dissociation Time." Journal of Chemical Theory and Computation 12 (6): 2983-89. https://doi.org/10.1021/acs.jctc.6b00277.

13. Casasnovas, Rodrigo, Vittorio Limongelli, Pratyush Tiwary, Paolo Carloni, and Michele Parrinello. 2017. "Unbinding Kinetics of a p38 MAP Kinase Type II Inhibitor from Metadynamics Simulations." Journal of the American Chemical Society 139 (13): 4780-88. https://doi.org/10.1021/jacs.6b12950.

14. Mollica, Luca, Isabelle Theret, Mathias Antoine, Françoise Perron-Sierra, Yves Charton, Jean-Marie Fourquez, Michel Wierzbicki, et al. 2016. "Molecular Dynamics Simulations and Kinetic Measurements to Estimate and Predict Protein-Ligand Residence Times." Journal of Medicinal Chemistry 59 (15): 7167-76. https://doi.org/10.1021/acs.jmedchem.6b00632.

15. Cuenda, Ana, and Simon Rousseau. 2007. "p38 MAP-Kinases Pathway Regulation, Function and Role in Human Diseases." Biochimica et Biophysica Acta (BBA) - Molecular Cell Research, Mitogen-Activated Protein Kinases: New Insights on Regulation, Function and Role in Human Disease, 1773 (8): 1358-75. https://doi.org/10.1016/j.bbamcr.2007.03.010.

16. Bosc, Nicolas, Berthold Wroblowski, Samia Aci-Sèche, Christophe Meyer, and Pascal Bonnet. 2015. "A Proteometric Analysis of Human Kinome: Insight into Discriminant Conformation-Dependent Residues." ACS Chemical Biology 10 (12): 2827-40. https://doi.org/10.1021/acschembio.5b00555.

17. Redhead, M., R. Satchell, V. Morkūnaitè, D. Swift, V. Petrauskas, E. Golding, S. Onions, D. Matulis, and J. Unitt. 2015. "A Combinatorial Biophysical Approach; FTSA and SPR for Identifying Small Molecule Ligands and PAINs." Analytical Biochemistry 479 (June): 63-73. https://doi.org/10.1016/j.ab.2015.03.013.

18. Regan, John, Christopher A. Pargellis, Pier F. Cirillo, Thomas Gilmore, Eugene R. Hickey, Gregory W. Peet, Alfred Proto, Alan Swinamer, and Neil Moss. 2003. "The Kinetics of Binding to p38MAP Kinase by Analogues of BIRB 796." Bioorganic \& Medicinal Chemistry Letters 13 (18): 3101-4.

19. Suárez, Ernesto, Adam J. Pratt, Lillian T. Chong, and Daniel M. Zuckerman. 2016. "Estimating First- passage Time Distributions from Weighted Ensemble Simulations and non- Markovian Analyses." Protein Science : A Publication of the Protein Society 25 (1): 67-78. https://doi.org/10.1002/pro.2738.

20. Hummer, Gerhard, and Attila Szabo. 2003. "Kinetics from Nonequilibrium Single-Molecule Pulling Experiments." Biophysical Journal 85 (1): 5-15.

21. Roux, B., 1995. The calculation of the potential of mean force using computer simulations. Computer Physics Communications 91, 275-282. https://doi.org/10.1016/0010-4655(95)00053-I

22. Bell, G. I. 1978. "Models for the Specific Adhesion of Cells to Cells." Science (New York, N.Y.) 200 (4342): 618-27.

23. Evans, E, and K Ritchie. 1997. "Dynamic Strength of Molecular Adhesion Bonds." Biophysical Journal 72 (4): 1541-55.

24. Lee, Chih-Kung, Yu-Ming Wang, Long-Sun Huang, and Shiming Lin. 2007. "Atomic Force Microscopy: Determination of Unbinding Force, off Rate and Energy Barrier for Protein-Ligand Interaction." Micron (Oxford, England: 1993) 38 (5): 446-61. https://doi.org/10.1016/j.micron.2006.06.014.

25. Björnham, Oscar, Jeanna Bugaytsova, Thomas Borén, and Staffan Schedin. 2009. "Dynamic Force Spectroscopy of the Helico- 
bacter Pylori BabA-Lewis B Binding." Biophysical Chemistry 143 (12): 102-5. https://doi.org/10.1016/j.bpc.2009.03.007. 
Path investigation, free energy estimation, kinetic and residence time prediction from unbinding simulations and transition state theory, with high level of correlation between estimated values and experimental measurements.
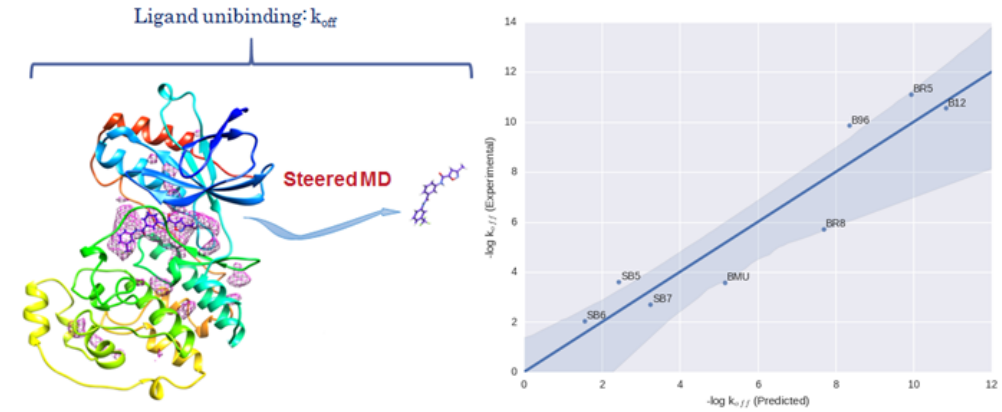

A. Braka, S. Aci-

Sèche, N. Garnier, $P$. Bonnet *

Page No. - Page No.

Residence Time

Prediction of Type 1

and 2 Kinase Inhibi-

tors from Unbinding

Simulations 